\title{
Gender and Fear of COVID-19 in a Cuban Population Sample
}

\section{Yunier Broche-Pérez ${ }^{1,2}$ (D) Zoylen Fernández-Fleites ${ }^{1,2}$ • Elizabeth Jiménez-Puig ${ }^{1,2}$. Evelyn Fernández-Castillo ${ }^{1,2} \cdot$ Boris C. Rodríguez-Martin ${ }^{3}$}

Published online: 12 June 2020

(C) Springer Science+Business Media, LLC, part of Springer Nature 2020

\begin{abstract}
The COVID-19 pandemic is a global health emergency that could potentially have a serious impact on public health. Fear has been one of the most frequent psychological reaction in the population during the current pandemic. The aim of this study was to compare fear of COVID-19 between genders and to examine whether the differences between genders may be predictors of fear of COVID-19 scores. A cross-sectional webbased survey design was adopted. The sample comprised 772 Cuban participants. The Fear of COVID-19 Scale was used to explore fear reactions in the sample. An independent-samples $t$ test was conducted to compare the fear of COVID-19 scores between genders, and multinomial logistic regression was modeling to identify variables independently associated with fear of coronavirus. In our sample, on average, female participants experienced significantly greater fear of COVID-19 than men. The gender of participants significantly predicted the level of fear of COVID-19. Being female was a predictor of medium and high levels of fear of COVID-19. The odds of a female with middle fear levels compared to low fear was 3.13 times more than for a male, and the odds of a female with high fear levels compared to low fear was 3.45 times more than for a male. Our results corroborate international research that indicate a greater psychological vulnerability in women during the COVID-19 pandemic. This result points to the need to design interventions that reduce the negative impact of the current outbreak on women's mental health.
\end{abstract}

Keywords Fear $\cdot$ Fear of COVID-19 $\cdot$ Gender $\cdot$ Psychological impact

Yunier Broche-Pérez

yunierbp@uclv.edu.cu; yunierbroche@gmail.com

1 Psychology Department, Universidad Central "Marta Abreu" de Las Villas, Km 5 1⁄2,, 54830 Santa Clara, Villa Clara, Cuba

2 CognitiON (Cuban Initiative on Cognitive Health), Santa Clara, Cuba

3 Fundación RECAL, Madrid, Spain 


\section{Background}

The COVID-19 pandemic is a global health emergency that could potentially have a serious impact on public health (Xiang et al. 2020). The outbreak was first revealed in late December 2019 when clusters of pneumonia cases of unknown etiology were found in the city of Wuhan of Hubei Province, in China (Wang et al. 2020). To date (27/5/20), there have been confirmed cases of COVID-19 in 217 countries, with a total of 5,556,679 infected people and a mortality of 351,866 cases (World Health Organization 2020). In Cuba, 1983 people have been diagnosed with the disease so far and 82 have died (Ministry of Public Health 2020).

The fast spread of the disease throughout the world, and the measures to contain it, is leading to mental health problems such as stress, anxiety, depressive symptoms, insomnia, denial, anger, and fear globally (Brooks et al. 2020; Galea et al. 2020; Qiu et al. 2020; Torales et al. 2020; Xiang et al. 2020). Fear has been one of the most frequent psychological reaction in the population during the current pandemic (Wang et al. 2020). This reaction is understandable as people are concerned for their health (Lin 2020). According to de Hoog et al. (2008), fear is defined as an unpleasant emotional state that is triggered by the perception of threatening stimuli. A more pragmatic definition is proposed by Ralph (2013) considering fear as an intervening variable between set of context-dependent stimuli and suites of behavioral response.

Fear can be beneficial during the COVID-19 pandemic, or it can also be detrimental to mental and physical health. Experiencing fear can increase the risk perception, promoting the protective behaviors (washing hands and maintaining physical distance, etc.). For example, a study (Harper et al. 2020) suggest that individuals engage more in preventive behaviors when they perceive the threat as severe. In the case of COVID-19, perceived threat could act as a motivational factor to perform a behavior that facilitates COVID-19 prevention (Harper et al. 2020; Pakpour and Griffiths 2020). Recent evidence suggest that fear also promotes social presence in anticipation of seeking affection, acceptance, and social information, promoting purchase behavior towards personal protective equipment (Addo et al. 2020).

On the other hand, fear can have catastrophic results. With high levels of fear, individuals may not think clearly and rationally when reacting to COVID-19. Following the spread of COVID-19 from Wuhan, discrimination towards Chinese people has increased (Devakumar et al. 2020). In this sense, fear is a key ingredient for racism and xenophobia to thrive. Fear also increase the suicide risk. This year, on March, a 36-year-old Bangladeshi man committed suicide because he and the people in his village thought he was infected with COVID-19 (Mamun and Griffiths 2020). Unfortunately, the autopsy showed that the victim did not have the disease. Fear is also associated with high levels of anxiety in the general population and health care professionals (Lee 2020; Shanafelt et al. 2020), anger (Khosravi 2020), and depression (Ahorsu et al. 2020).

As has been suggested, it is important to carry new studies about fear, considering variables such as gender, age, education, ethnicity, and/or religiosity (Pakpour and Griffiths 2020).

For example, female gender is significantly associated with a greater psychological impact of the outbreak and higher levels of stress, anxiety, insomnia, perceived stress, adjustment disorder, and depression (Rossi et al. 2020; Wang et al. 2020). However, as far as we know, the relationship between fear of COVID-19 and gender has not been explored. The aim of this study was first to compare fear of COVID-19 between genders. The second purpose was to examine whether the differences between genders and social factors may be predictors of fear of COVID-19 scores. 


\section{Methods}

\section{Study Design and Participants}

A cross-sectional web-based survey design was adopted. Online consent was obtained from the participants. The survey was anonymous, and confidentiality of information was assured. Data on participants were collected between April 4 and May 27, 2020 using an online questionnaire spread throughout the internet. The survey was developed using the free software Google Forms ${ }^{\circledR}$. The target population was the general Cuban population. All Cuban citizens over 18 years were eligible. A total of 786 participants completed the questionnaire. Of the total sample, 14 participants were excluded because they were less than 18 years old. The final sample was made up of 772 participants. For this sample size, a power analysis was running (post hoc) using the G*Power software (version 3.1.9.2) (Faul et al. 2009). Considering the sample size, the analysis (two-tailed) showed a power of 0.82 .

\section{Measures}

\section{Demographic Information}

The demographic variables explored included the age, gender, and region of the country where the participant resided. Additionally, it was explored if the participant resided in a place with confirmed cases of COVID-19 ("yes", "no", "I don't know"), if he/she knew an infected person ("yes" or "no"), and if a member of family or friend has been confirmed with the disease ("yes" or "no").

\section{The Fear of COVID-19 Scale}

The Fear of COVID-19 Scale was recently developed by Ahorsu et al. (2020). The scale is made up of 7 items, grouped into a single-factor structure. According to Ahorsu et al. (2020), the Fear of COVID-19 Scale has good internal consistency, with a Cronbach alpha coefficient reported of 0.82. In Cuban population, the Cronbach alpha coefficient was 0.87 .

The participants indicate their level of agreement with the statements using a five-item Likert-type scale ("strongly disagree," "disagree," "neither agree nor disagree," "agree," and "strongly agree"). The minimum score possible for each question is 1 , and the maximum is 5 . A total score is calculated by adding up each item score (ranging from 7 to 35). The higher the score, the greater the fear of cororonavirus-19.

For this study, the original scale was translated following the suggestions of the International Test Commission (ITC) (International Test Commission 2017). The forward-backward translation method was applied to adapt the FCV-19S into Spanish (Cuba). Two independent translators translated (forward translation) the Fear of COVID-19 Scale into Spanish. In the back-translation process, both versions were translated back into English by a professional translator with experience in psychological research and by one bilingual neuropsychology professor (blind to the original version of the Fear of COVID-19 Scale). A panel of three experts suggest modifications and approved the final version (Appendix). Recently, the Fear of COVID-19 Scale was also validated for Italian sample (Soraci et al. 2020) and Bangla sample (Sakib et al. 2020). 


\section{Data Analysis}

The data was approved by the ethics committee of the Department of Psychology of the Universidad Central "Marta Abreu" de Las Villas. Informed consent was obtained for all patients for being included in the study.

The data were processed using SPSS/Windows, version 21. Descriptive statistics was used to explore participants' characteristics. An independent-samples $t$ test was conducted to compare the fear of COVID-19 scores between genders. The results were filtered using the Benjamini-Hochberg procedure for FDR correction (FDR < 0.05) (Benjamini and Hochberg 1995). Effect sizes were calculated using Cohen's $d$. Cohen classifies 0.2 as a small effect, 0.5 as a medium effect, and 0.8 as a large effect (Cohen 1988). A model of main effects for multinomial logistic regression was modeling to identify variables independently associated with fear of coronavirus.

\section{Results}

\section{Characteristics of the Sample}

The mean age of participants $(n=772)$ was 36 years $(\mathrm{SD} \pm 14.61)$, with a range between 18 and 82 years old. In the sample, $569(73.7 \%)$ participants were females and 203 males $(26.3 \%)$.

A total of 515 participants $(66.7 \%)$ live in areas where there are confirmed cases of COVID-19. On the other hand, $78.8 \%(n=608)$ of the participants do not directly meet any patient infected with the disease, while $41(5.3 \%)$ participants have a close relative or friend positive to COVID-19. The central provinces of the country were the most represented $(n=$ $704,91.2 \%)$.

\section{Comparisons Between Genders on the Fear of Coronavirus-19 Scale}

An independent-samples $t$ test was conducted to compare fear of COVID-19 scores for males and females. On average, female participants experienced significantly greater fear of COVID19 than men. As shown in Table 1, except for item 3, differences were found in all scale items. For item 5, and Total Score, the magnitude of the differences in the means was medium $(d>$ 0.5 ). The effect size of the differences in the means for item 1 , item 2 , item 4 , item 6 , and item 7 was small $(d>0.2)$.

\section{Association Between Gender of Participants with Levels of Fear of Coronavirus-19}

High, middle, and low levels of fear of COVID-19 were established according to sample percentiles. A total of 212 participants (27.5\%) showed low levels of fear of COVID-19, 384 participants (49.7\%) middle levels of fear, and 175 participants $(22.7 \%)$ high levels of fear.

The association between gender of participants with levels of fear was investigated using multinomial logistic regression. Preliminary analyses were performed to ensure no violation of the assumptions of linearity, independence of errors, and multicollinearity (Field 2012). As shown in Table 2, the gender of participants significantly predicted whether they had middle or low fear of COVID-19, $b=-1.145$, Wald ${ }_{X}^{2}(1)=35.34, p<0.001$. According to the effect of 
Table 1 Comparisons between genders on the Fear of Coronavirus-19 Scale

\begin{tabular}{|c|c|c|c|c|c|c|}
\hline & $\begin{array}{l}\text { Male }(n=203) \\
\text { Mean }(S D)\end{array}$ & $\begin{array}{l}\text { Female }(n=569) \\
\text { Mean }(\mathrm{SD})\end{array}$ & $t$ & $p$ & $\begin{array}{l}\mathrm{B}-\mathrm{H} \\
p \text { value }\end{array}$ & $d$ \\
\hline $\begin{array}{l}\text { Item } 1 \text {. I am most afraid of } \\
\text { coronavirus } 19 .\end{array}$ & $2.93(1.77)$ & $3.66(1.61)$ & 5.35 & $<0.001$ & 0.001 & 0.44 \\
\hline $\begin{array}{l}\text { Item } 2 . \text { It makes me } \\
\text { uncomfortable to think } \\
\text { about coronavirus- } 19 \text {. }\end{array}$ & $3.71(1.65)$ & $4.24(1.25)$ & 4.70 & $<0.001$ & 0.001 & 0.38 \\
\hline $\begin{array}{l}\text { Item } 3 . \text { My hands become } \\
\text { clammy when I think about } \\
\text { coronavirus- } 19 \text {. }\end{array}$ & $1.56(1.24)$ & $1.62(1.25)$ & 0.56 & 0.576 & 0.05 & 0.04 \\
\hline $\begin{array}{l}\text { Item } 4 . \text { I am afraid of losing my } \\
\text { life because of coronavirus- } 19 \text {. }\end{array}$ & $2.79(1.76)$ & $3.12(1.76)$ & 2.30 & 0.021 & 0.027 & 0.18 \\
\hline $\begin{array}{l}\text { Item } 5 \text {. When watching news and } \\
\text { stories about coronavirus-19 on } \\
\text { social media, I become } \\
\text { nervous or anxious. }\end{array}$ & $2.91(1.67)$ & $3.91(1.42)$ & 8.14 & $<0.001$ & 0.001 & 0.60 \\
\hline $\begin{array}{l}\text { Item } 6 \text {. I cannot sleep because I'm } \\
\text { worrying about getting coronavirus- } 19 \text {. }\end{array}$ & $1.77(1.36)$ & $2.28(1.61)$ & 3.98 & $<0.001$ & 0.001 & 0.33 \\
\hline $\begin{array}{l}\text { Item } 7 . \text { My heart races or palpitates } \\
\text { when I think about getting } \\
\text { coronavirus-19 }\end{array}$ & $2.31(1.61)$ & $3.09(1.76)$ & 5.49 & $<0.001$ & 0.001 & 0.45 \\
\hline Total score & $17.9(8.05)$ & $21.9(6.9)$ & 6.60 & $<0.001$ & 0.001 & 0.55 \\
\hline
\end{tabular}

$B-H$ Benjamini-Hochberg

male compared with female, the odds ratio indicates that as sex changes from male to female, the change in the odds of having middle fear levels compared to low fear is 0.32 . In other words, the odds of a female with middle fear levels compared to low fear are $1 / .32=3.13$ times more than for a male. The gender also significantly predicted whether they had high fear, $b=-$ 1.25 , Wald $X^{2}(1)=26.63, p<0.05$. The odds of a woman with middle fear compared to low fear are $1 / .29=3.45$ times more than for a man.

Table 2 Gender and its association with fear to COVID-19

\begin{tabular}{|c|c|c|c|c|}
\hline & \multirow[b]{2}{*}{$\mathrm{B}(\mathrm{SE})$} & \multicolumn{3}{|c|}{$95 \%$ CI for odds ratio } \\
\hline & & Lower & OR & Upper \\
\hline \multicolumn{5}{|c|}{ Middle vs. low feara } \\
\hline \multicolumn{5}{|l|}{ Gender } \\
\hline $\begin{array}{l}\text { Male } \\
\text { Female }\end{array}$ & $-1.14(0.19) * * *$ & 0.22 & 0.32 & 0.47 \\
\hline \multicolumn{5}{|c|}{ High vs. low fear ${ }^{a}$} \\
\hline Intercept & $-.73(0.26) * *$ & & & \\
\hline \multicolumn{5}{|l|}{ Gender } \\
\hline $\begin{array}{l}\text { Male } \\
\text { Female }\end{array}$ & $-1.25(0.24) * * *$ & 0.18 & 0.29 & 0.46 \\
\hline
\end{tabular}

CI confidence intervals

$* p<0.05 ; * * p<0.01 ; * * * p<0.001$

a Reference category 


\section{Discussion}

The aim of this study was, first, to compare fear of COVID-19 between genders. The second purpose was to examine whether the differences between genders and social factors may be predictors of fear of COVID-19 scores. The main findings can be summarized as follows: (1) fear of COVID-19 was more severe in the female group than in the male group, and (2) the gender of participants significantly predicted the level of fear of COVID-19.

In particular, in our sample, being female is a predictor of medium and high levels of fear of COVID-19. Globally, the study demonstrated higher level of fear of COVID-19 in females.

This is consistent with other reports that have shown greater psychological vulnerability in women compared to men during the COVID-19 pandemic (Liu et al. 2020; Rossi et al. 2020; Wang et al. 2020). For example, in a study conducted by Wang et al. (2020), female gender was found to be a predictor of the negative psychological impact of the COVID-19 outbreak. This study included 1210 respondents from 194 cities in China. According to authors, females suffered a greater psychological impact of the outbreak as well as higher levels of stress, anxiety, and depression.

Another study explored the prevalence and predictors of post-traumatic stress symptoms (PTSS) during the COVID-19 outbreak in China (Liu et al. 2020). In the study, women reported significantly higher PTSS in the domains of re-experiencing, negative alterations in cognition or mood, and hyper-arousal. According to the authors, the differences found could be related to the fact that women show more reactivity than men in neural networks associated with fear and arousal responses and greater differential conditioned skin conductance responses to aversive stimuli.

In Italy, results are also reported that suggest a greater psychological vulnerability associated with COVID-19 in a woman (Rossi et al. 2020). According to the study authors, female gender was significantly associated with a greater psychological impact of the outbreak and higher levels of stress, anxiety, insomnia, perceived stress, adjustment disorder, and depression.

That females experience more fear than male during COVID-19 may be related to several factors. Some factors may be immediate and others have long-term implications. Fear may result from anticipating a negative impact of the disease on one's health and the health of close family and friends. On the other hand, closures of schools and daycare centers have massively increased child care needs, which has a particularly large impact on working mothers (Alon et al. 2020).

Moderate levels of fear can also have a positive impact and motivate healthy behaviors during the outbreak. For example, the differences found in our study between genders could be directly related to risk perception (RP). A recent study reported that gender was the only sociodemographic predictor variable of risk perception levels in a sample of 6991 participants, where being a female was a predictor of high levels of RP (Dryhurst et al. 2020). A moderate fear of COVID-19 could increase the perception of risk against the disease, promoting preventive behaviors (Harper et al. 2020; Pakpour and Griffiths 2020).

\section{Limitations and Future Directions}

This research is not without limitations. First, this is a cross-sectional study so it is difficult to accurately elucidate causal relationships between gender and fear of COVID-19. Additional longitudinal studies in this field are essential in the future. We also consider that the sample is relatively small, considering that it is an online study. This limitation could be caused by the 
difficulties of internet connectivity in Cuba. On the other hand, our study did not control which participants were in quarantine zones and which were not. Quarantine can amplify negative psychological reactions to COVID-19 (Rossi et al. 2020). In Cuba, most places have confirmed cases of COVID-19 exist, physical distancing measures are implemented, and only in specific places have mandatory quarantines been implemented. In future studies, the relationship between fear of COVID-19 and other variables such as education level, risk perception, anxiety, depression, and coping styles should be explored. These studies will make it possible to verify the interactions between fear of COVID-19 and other psychological reactions.

\section{Conclusions}

In conclusion, our findings show that fear of COVID-19 was more severe in the female participants than in male participants, and also, the gender of participants is a predictor of the level of fear of COVID-19 in our sample. Those results are relevant especially in the Cuban context. First, the study suggests that, in our sample, belonging to female gender is a predictor for experiencing medium and high levels of fear. This result points to the need to design interventions that reduce the negative impact of the current outbreak on women's mental health. Additionally, our results corroborate international research that indicate a greater psychological vulnerability in women during the COVID-19 pandemic. It is very important to continue monitoring the psychological reactions of women during the outbreak. This evidence could help policy-makers design evidenced-based intervention strategies, with special emphasis in the most vulnerable groups.

Authors' Contributions Study planning: YBP. Study plan validation: All authors. Data interpretation: All authors. Data analysis: YBP and ZFF. First draft: YBP. Final approval: All authors.Data AvailabilityData is available on https://doi.org/10.13140/RG.2.2.22836.88964

\section{Compliance with Ethical Standards}

All procedures followed were in accordance with the ethical standards of the responsible committee on human experimentation (institutional and national) and with the Helsinki Declaration of 1975, as revised in 2000. Informed consent was obtained for all patients for being included in the study.

Conflict of Interest The authors declare that they have no conflict of interest.

\section{Appendix. Fear of Coronavirus-19 Scale (Spanish (Cuban) version)}

Orientaciones: Indique el nivel de acuerdo con las siguientes proposiciones empleando la siguiente escala: (5) Completamente de acuerdo, (4) De acuerdo, (3) Ni de acuerdo, ni en desacuerdo, (2) En desacuerdo y (1) Completamente en desacuerdo.

1. Siento mucho miedo del COVID-19 (I am most afraid of coronavirus-19)

2. Me hace sentir incómodo pensar acerca del COVID-19 (It makes me uncomfortable to think about coronavirus-19)

3. Mis manos sudan cuando pienso sobre el COVID-19 (My hands become clammy when I think about coronavirus-19) 
4. Tengo miedo a perder mi vida por el COVID-19 (I am afraid of losing my life because of coronavirus-19)

5. Cuando veo las noticias y escucho historias sobre el COVID-19 en los medios de comunicación me pongo nervioso y ansioso (When watching news and stories about coronavirus-19 on social media, I become nervous or anxious)

6. No puedo dormir porque me preocupo por la posibilidad de contagiarme con COVID-19 (I cannot sleep because I'm worrying about getting coronavirus-19)

7. Mi corazón se acelera cuando pienso que puedo contagiarme con el COVID-19 (My heart races or palpitates when I think about getting coronavirus-19)

\section{References}

Addo, P. C., Jiaming, F., Kulbo, N. B., \& Liangqiang, L. (2020). COVID-19: fear appeal favoring purchase behavior towards personal protective equipment. The Service Industries Journal., 40, 471-490. https://doi. org/10.1080/02642069.2020.1751823.

Ahorsu, D. K., Lin, C.-Y., Imani, V., Safari, M., Griffiths, M. D., \& Pakpour, A. H. (2020). The Fear of COVID19 Scale: development and initial validation. International Journal of Mental Health and Addiction. https://doi.org/10.1007/s11469-020-00270-8.

Alon, T. M., Doepke, M., Olmstead-Rumsey, J., \& Tertilt, M. (2020). The impact of COVID-19 on gender equality. National Bureau of Economic Research. https://doi.org/10.3386/w26947.

Benjamini, Y., \& Hochberg, Y. (1995). Controlling the false discovery rate: a practical and powerful approach to multiple resting. Journal of the Royal Statistical Society. Series B (Methodological), 57(1), 289-300.

Brooks, S. K., Webster, R. K., Smith, L. E., Woodland, L., Wessely, S., Greenberg, N., \& Rubin, G. J. (2020). The psychological impact of quarantine and how to reduce it: rapid review of the evidence. Lancet, 395, 912-920. https://doi.org/10.1016/S0140-6736(20)30460-8.

Cohen, J. (1988). Statistical power analysis for the behavioral sciences (2nd ed.). Acad Press.

de Hoog, N., Stroebe, W., \& de Wit, J. B. (2008). The processing of fear-arousing communications: how biased processing leads to persuasion. Social Influence, 3(2), 84-113. https://doi.org/10.1080/15534510802185836.

Devakumar, D., Shannon, G., Bhopal, S., \& Abubakar, I. (2020). Racism and discrimination in COVID-19 responses. The Lancet, 395, 1194. https://doi.org/10.1016/S0140-6736(20)30792-3.

Dryhurst, S., Schneider, C. R., Kerr, J., Freeman, A. L. J., Recchia, G., van der Bles, A. M., Spiegelhalter, D., \& van der Linden, S. (2020). Risk perceptions of COVID-19 around the world. Journal of Risk Research., 113. https://doi.org/10.1080/13669877.2020.1758193.

Faul, F., Erdfelder, E., Buchner, A., \& Lang, A.-G. (2009). Statistical power analyses using G*Power 3.1: tests for correlation and regression analyses. Behavior Research Methods, 41(4), 1149-1160. https://doi. org/10.3758/BRM.41.4.1149.

Field, A. (2012). Discovering statistics using SPSS (and sex and drugs and rock 'n' roll) (Cuarta ed.). SAGE Publications.

Galea, S., Merchant, R. M., \& Lurie, N. (2020). The mental health consequences of COVID-19 and physical distancing: the need for prevention and early intervention. JAMA Internal Medicine, E1-E2. https://oi. org/10.1001/jamainternmed.2020.1562.

Harper, C. A., Satchell, L. P., Fido, D., \& Latzman, R. D. (2020). Functional fear predicts public health compliance in the COVID-19 pandemic. International Journal of Mental Health and Addiction. https://doi.org/10.1007/s11469-020-00281-5.

International Test Commission. (2017). ITC guidelines for translating and adapting tests (second edition). International Journal of Testing., 18, 101-134. https://doi.org/10.1080/15305058.2017.1398166.

Khosravi, M. (2020). Perceived risk of COVID-19 pandemic: the role of public worry and trust. Electron J Gen Med, 17(4), em203. https://doi.org/10.29333/ejgm/7856.

Lee, S. A. (2020). Coronavirus Anxiety Scale: a brief mental health screener for COVID-19 related anxiety. Death Studies., 44, 393-401. https://doi.org/10.1080/07481187.2020.1748481.

Lin, C. Y. (2020). Social reaction toward the 2019 novel coronavirus (COVID-19). Social Health and Behavior, 3, 1-2. https://doi.org/10.4103/SHB.SHB_11_2. 
Liu, N., Zhang, F., Wei, C., Jia, Y., Shang, Z., \& Sun, L. (2020). Prevalence and predictors of PTSS during 322 COVID-19 outbreak in China hardest-hit areas: gender differences matter. Psychiatry Research, 112921, 112921. https://doi.org/10.1016/j.psychres.2020.112921.

Mamun, M. A., \& Griffiths, M. D. (2020). First COVID-19 suicide case in Bangladesh due to fear of COVID-19 and xenophobia: possible suicide prevention strategies. Asian Journal of Psychiatry, 61, 102073. https://doi. org/10.1016/j.ajp.2020.102073.

Ministry of Public Health. (2020). Closing report of may 27 at 12 midnight. Ministry of Public Health https://salud.msp.gob.cu/?cat=839.

Pakpour, A. H., \& Griffiths, M. D. (2020). The fear of COVID-19 and its role in preventive behaviors. Journal of Concurrent Disorders 2 (1), 58-63.

Qiu, J., Shen, B., Zhao, M., Wang, Z., Xie, B., \& Xu, Y. (2020). A nationwide survey of psychological distress 331 among Chinese people in the COVID-19 epidemic: Implications and policy recommendations. General Psychiatry, 33, 19-21. https://doi.org/10.1136/gpsych-2020-100213.

Ralph, A. (2013). The biology of fear. Current Biology, 23(2), R79-R93. https://doi.org/10.1016/j. cub.2012.11.055.

Rossi, R., Socci, V., Talevi, D., Mensi, S., Niolu, C., Pacitti, F., Di MArco, A., Rossi, A., Siracusano, A., \& Di Lorenzo, G. (2020). COVID-19 pandemic and lockdown measures impact on mental health among the general population in Italy. An $\mathrm{N}=18147$ web-based 2 survey. medRxiv. https://doi.org/10.1101 /2020.04.09.20057802.

Sakib, N., Mamun, M. A., Bhuiyan, A., Hossain, S., Mamun, F. A., Hosen, I., Adbdullah, A. H., Sarker, A., Mohiuddin, S., Rayhan, I., Hossain, M., Sikder, T., Gozal, D., Muhit, M. A., Islam, S. M. S., Griffiths, M. D., \& Pakpour, A. H. (2020). Psychometric validation of the Bangla Fear of COVID-19 Scale: confirmatory factor analysis and Rasch analysis. International Journal of Mental Health and Addiction. https://oi. org/10.1007/s11469-020-00289-x.

Shanafelt, T., Ripp, J., \& Trockel, M. (2020). Understanding and addressing sources of anxiety among health care professionals during the COVID-19 pandemic. JAMA Internal Medicine, 323, E2-E1. https:/doi. org/10.1001/jama.2020.5893.

Soraci, P., Ferrari, A., Abbiati, F. A., Del Fante, E., De Pace, R., Urso, A., \& Griffiths, M. D. (2020). Validation and psychometric evaluation of the Italian version of the fear of COVID-19 scale. International Journal of Mental Health and Addiction. https://doi.org/10.1007/s11469-020-00277-1.

Torales, J., O’Higgins, M., Castaldelli-Maia, J. M., \& Ventriglio, A. (2020). The outbreak of COVID-19 coronavirus and its impact on global mental health. International Journal of Social Psychiatry, 1-4. https://doi.org/10.1177/0020764020915212.

Wang, C., Pan, R., Wan, X., Tan, Y., Xu, L., \& Ho, C. S. (2020). Immediate psychological responses and 347 associated factors during the initial stage of the 2019 coronavirus disease (COVID-19) epidemic among the 348 general population in China. International Journal of Environmental Research and Public Health, 17. https://doi.org/10.3390/ijerph17051729.

World Health Organization. (2020). Coronavirus disease (COVID-19) pandemic. World Health Organization https://www.who.int/emergencies/diseases/novel-coronavirus-2019.

Xiang, Y.-T., Yang, Y., Li, W., Zhang, L., Zhang, Q., \& Cheung, T. (2020). Timely mental health care for the 2019 novel coronavirus outbreak is urgently needed. The Lancet Psychiatry, 7, 228-229 Disponible en: 210.1016/S2215-0366(1020)30046-30048. https://doi.org/10.1016/S2215-0366(20)30046-8.

Publisher's Note Springer Nature remains neutral with regard to jurisdictional claims in published maps and institutional affiliations. 